This is the accepted manuscript made available via CHORUS. The article has been published as:

\title{
Coherent Spin Pumping in a Strongly Coupled Magnon- Magnon Hybrid System
}

Yi Li, Wei Cao, Vivek P. Amin, Zhizhi Zhang, Jonathan Gibbons, Joseph Sklenar, John Pearson, Paul M. Haney, Mark D. Stiles, William E. Bailey, Valentine Novosad, Axel Hoffmann, and Wei Zhang

Phys. Rev. Lett. 124, 117202 - Published 17 March 2020

DOI: 10.1103/PhysRevLett.124.117202 


\title{
Coherent spin pumping in a strongly coupled magnon-magnon hybrid system
}

\author{
Yi Li, ${ }^{1,2}$ Wei Cao, ${ }^{3}$ Vivek P. Amin, ${ }^{4,5}$ Zhizhi Zhang,,${ }^{2,6}$ Jonathan Gibbons, ${ }^{2}$ Joseph Sklenar, ${ }^{7}$ John Pearson, ${ }^{2}$ Paul \\ M. Haney, ${ }^{5}$ Mark D. Stiles, ${ }^{5}$ William E. Bailey, ${ }^{3,}{ }^{*}$ Valentine Novosad, ${ }^{2}$ Axel Hoffmann, ${ }^{2,} \dagger$ and Wei Zhang ${ }^{1,2}, \ddagger$ \\ ${ }^{1}$ Department of Physics, Oakland University, Rochester, MI 48309, USA \\ ${ }^{2}$ Materials Science Division, Argonne National Laboratory, Argonne, IL 60439, USA \\ ${ }^{3}$ Materials Science and Engineering, Department of Applied Physics and Applied Mathematics, \\ Columbia University, New York, New York 10027, USA \\ ${ }^{4}$ Maryland Nanocenter, University of Maryland, College Park, MD 20742, USA \\ ${ }^{5}$ Center for Nanoscale Science and Technology, National Institute \\ of Standards and Technology, Gaithersburg, Maryland 20899, USA \\ ${ }^{6}$ School of Optical and Electronic Information, Huazhong University of Science and Technology, Wuhan 430074, China \\ ${ }^{7}$ Department of Physics and Astronomy, Wayne State University, Detroit, MI 48202, USA
}

(Dated: January 10, 2020)

\begin{abstract}
We experimentally identify coherent spin pumping in the magnon-magnon hybrid modes of permalloy/yttrium iron garnet (Py/YIG) bilayers. By reducing the Py and YIG thicknesses, the strong interfacial exchange coupling leads to large avoided crossings between the uniform mode of Py and the spin wave modes of YIG enabling accurate determination of modification of the linewidths due to the dampinglike torque. We identify additional linewidth suppression and enhancement for the in-phase and out-of-phase hybrid modes, respectively, which can be interpreted as concerted dampinglike torque from spin pumping. Furthermore, varying the Py thickness shows that both the fieldlike and dampinglike couplings vary like $1 / \sqrt{t_{P y}}$, verifying the prediction by the coupled Landau-Lifshitz equations.
\end{abstract}

Coherent information processing has recently become an emerging topic for the post-CMOS electronics era $[1,2]$. In spintronics, exchange-induced magnetic excitations, called spin waves, or magnons [3, 4], are good candidates because information can be encoded by both the amplitude and the phase of spin waves. For example, the interference of coherent spin waves can be engineered for spin wave logic operations [5-7]; the coherent interaction of spin-torque oscillators leads to mutual synchronization [8-13], which can be applied in artificial neural networks $[14,15]$; and the coherent coupling between magnons and microwave cavities [16-22] opens up new opportunities for magnon-based quantum information science [23, 24].

Recently, strong coupling between two magnonic systems has enabled excitations of forbidden spin wave modes [25-27] and high group velocity of propagating spin waves $[28,29]$. The coupling is dominated by the exchange interaction at the interface of the magnetic bilayers, providing a new pathway to coherently transfer magnon excitations between two magnetic systems possessing distinctive properties: from conductor to insulator, from uniform to nonuniform mode and from highdamping to low-damping systems. However, the underlying physical mechanisms of the coupling are still not fully understood. First, what are the key parameters that dictate the coupling efficiency and enable one to reach the strong-coupling regime? Second, with the interfacial exchange coupling acting as a fieldlike torque, is there a dampinglike torque associated with spin pumping [3033]? To resolve both questions, large separations of the two hybrid modes are required in order to quantitatively analyze the coupling mechanism. The second question is also important for optimizing the coherence of spin wave transfer in hybrid systems. Furthermore, the parasitic effect on the incoherent spin current from the conduction band is absent [34-36] by using magnetic insulators such as yttrium iron garnet $\left(\mathrm{Y}_{3} \mathrm{Fe}_{5} \mathrm{O}_{12}\right.$, YIG) $[29,37,38]$, which facilitates the study of spin pumping coherency.

In this work, we study YIG/permalloy $\left(\mathrm{Ni}_{80} \mathrm{Fe}_{20}, \mathrm{Py}\right)$ bilayers. By using much thinner YIG and Py films than studied in previous works [25, 27], we achieve an exchange-induced separation of the two hybrid modes much larger than their linewidths, allowing us to study the evolution of their linewidths in the strong coupling regime. We find a pronounced suppression of the total linewidth for in-phase hybrid modes and a linewidth enhancement for out-of-phase hybrid modes. The linewidths can be understood from the Landau-LifshitzGilbert (LLG) equation with interfacial exchange coupling and mutual spin pumping, which provide the fieldlike and dampinglike interlayer coupling torques, respectively. Furthermore, the thickness dependence of the two coupling strengths agrees with the modeling of coupled LLG equations with mutual spin pumping. The sign of the fieldlike torque also reconfirms that the YIG and Py are coupled antiferromagnetically [25]. Our results provide important insights for improving the coupling strength and coherence in magnon-magnon hybrid systems and pave the way for coherent information processing with exchange coupled magnetic heterostructures.

The samples consist of YIG(100 nm)/Py $\left(t_{\mathrm{Py}}\right)$ bilayers where $t_{\mathrm{Py}}$ varies from $5 \mathrm{~nm}$ to $60 \mathrm{~nm}$. YIG(100 nm) films were deposited by magnetron sputtering from a YIG target onto $\mathrm{Gd}_{3} \mathrm{Ga}_{5} \mathrm{O}_{12}(111)$ substrates and annealed in air at $850^{\circ} \mathrm{C}$ for $3 \mathrm{hrs}$ to reach low-damping characteristics [39]. Before the deposition of Py films on top of YIG, 

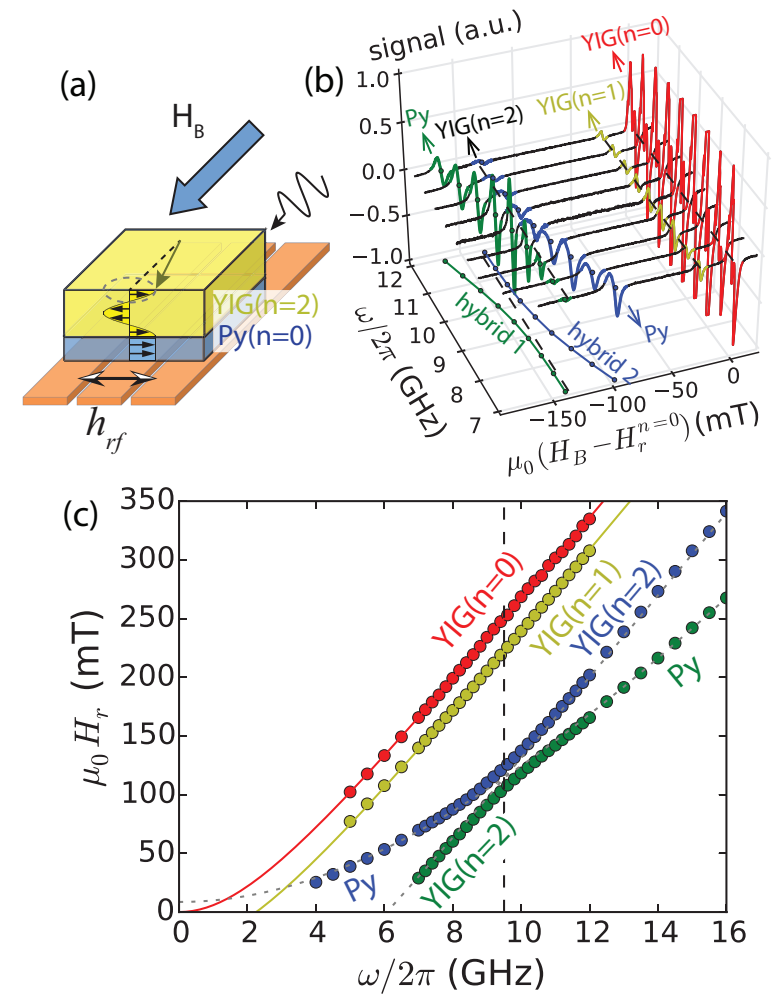

FIG. 1. (a) Illustration of the magnetization excitations in the Py/YIG bilayers with a coplanar waveguide. (b) Lineshapes of the Py $(9 \mathrm{~nm}) / \mathrm{YIG}(100 \mathrm{~nm})$ sample for the first three resonance modes of YIG and the uniform mode of Py. The field axis is shifted so that the resonance field of the $\mathrm{YIG}(\mathrm{n}=0)$ mode is zero. (c) Unshifted evolution of the four modes in (b). Curves show the fits as uncoupled modes. The vertical dashed line denotes where the $\operatorname{YIG}(n=2)$ and $\operatorname{Py}(n=0)$ modes cross on the frequency axis at $\omega_{c} / 2 \pi=9.4 \mathrm{GHz}$.

the YIG surfaces were ion milled in-situ for one minute in order to enable good exchange coupling between Py and YIG [40]. For each Py thickness, one additional, reference Py film was deposited on a $\mathrm{Si} / \mathrm{SiO}_{2}$ substrate during the same deposition.

The hybrid magnon dynamics were characterized by broad-band ferromagnetic resonance on a coplanar waveguide (Fig. 1a). An in-plane magnetic field $H_{B}$ saturates both the YIG and Py magnetizations. Their Kittel modes, which describe spatially uniform magnetization precession, are formulated as $\omega^{2} / \gamma^{2}=\mu_{0}^{2} H_{r}\left(H_{r}+M_{s}\right)$, where $\omega$ is the mode frequency, $\gamma / 2 \pi=\left(g_{\text {eff }} / 2\right) \times$ $27.99 \mathrm{GHz} / \mathrm{T}$ is the gyromagnetic ratio, $H_{r}$ is the resonance field and $M_{s}$ is the magnetization [41]. For YIG, the spatially nonuniform perpendicular standing spin wave (PSSW) modes can be also measured. An effective exchange field $H_{e x}$ will lower the resonance field as $\mu_{0} H_{e x}(k)=\left(2 A_{e x} / M_{s}\right) k^{2}$, where $A_{e x}$ is the exchange stiffness, $k=n \pi / t, n$ labels the index of PSSW modes, and $t$ is the film thickness [42].

Fig. 1(b) shows the line shapes of the resonance fields for the first three resonance modes of YIG $(n=0,1$, $2)$ and the Py uniform mode $(n=0)$ measured for $t_{\mathrm{Py}}=9 \mathrm{~nm}$. For illustration, the YIG $(n=0)$ resonance is shifted to zero field. An avoided crossing is clearly observed when the Py uniform mode is degenerate with the YIG $(n=2)$ mode. This is due to the exchange coupling at the YIG/Py interface [25-27] providing a fieldlike coupling torque. Both two YIGPy hybrid modes are strongly excited because the energy of the Py uniform mode is coherently transferred to the YIG PSSW modes through the interface [25]. The full-range frequency dependence of the extracted resonance field are plotted in Fig. 1(c). To analyze the two hybrid modes, we analyze our results with two independent Lorentzians because it facilitates a transparent physical picture and the fit lineshapes agree well with our measurements. The modes crossing happens at $\omega_{c} / 2 \pi=9.4 \mathrm{GHz}$ (black dashed line), which corresponds to the minimal resonance separation of the two hybrid modes. Fitting to the Kittel equation, we extract $\mu_{0} M_{s}^{\mathrm{YIG}}=0.21 \mathrm{~T}, \mu_{0} M_{s}^{\mathrm{Py}}=0.86 \mathrm{~T}$. From the exchange field offset as shown in Fig. 1(b), an exchange stiffness $A_{e x}=2.6 \mathrm{pJ} / \mathrm{m}$ is calculated for YIG, which is similar to previous reports [43].

The avoided crossing can be fitted to a phenomenological model of two coupled harmonic oscillators, as previously shown in magnon polaritons $[16-18,20]$ :

$$
\mu_{0} H_{c}^{ \pm}=\mu_{0} \frac{H_{r}^{\mathrm{YIG}}+H_{r}^{\mathrm{Py}}}{2} \pm \sqrt{\left(\mu_{0} \frac{H_{r}^{\mathrm{YIG}}-H_{r}^{\mathrm{Py}}}{2}\right)^{2}+g_{c}^{2}}
$$

where $H_{r}^{\mathrm{YIG}(\mathrm{Py})}$ is the resonance field of YIG $(\mathrm{Py})$, and $g_{c}$ is the interfacial exchange coupling strength. $H_{r}^{\mathrm{YIG}}$ and $H_{r}^{\mathrm{Py}}$ are both functions of frequency and are equal at $\omega_{c}$. Note that for in-plane biasing field the resonance field is nonlinear to the excitation frequency. This nonlinearity will be accounted in the analytical reproduction of Eq. 1. The fitting yields $g_{c}=8.4 \mathrm{mT}$ for $t_{\mathrm{Py}}=9 \mathrm{~nm}$.

Next, we focus on the linewidths of the YIG-Py hybrid modes for the study of the dampinglike torque. Fig. 2(a) shows the line shape of the two hybrid modes for $t_{\mathrm{Py}}=7.5 \mathrm{~nm}$ at $\omega_{c}$. These two eigenmodes correspond to the in-phase and out-of-phase magnetization precession of Py and YIG with the same weight, so they should yield the same total intrinsic damping. Nevertheless, a significant linewidth difference is observed, with the extracted full-width-half-maximum linewidth $\mu_{0} \Delta H_{1 / 2}$ varying from $3.5 \mathrm{mT}$ for the lower field resonance to $8.0 \mathrm{mT}$ for the higher field resonance. Fig. 2(b) shows the full-range evolution of linewidth. Compared with the dotted lines which are the linear extrapolations of the YIG $(n=2)$ and Py linewidths, the linewidth of the higher-field hybrid mode (blue circles) exceeds the Py linewidth and the linewidth of the lower-field hybrid mode (green circles) reduces below the YIG linewidth 

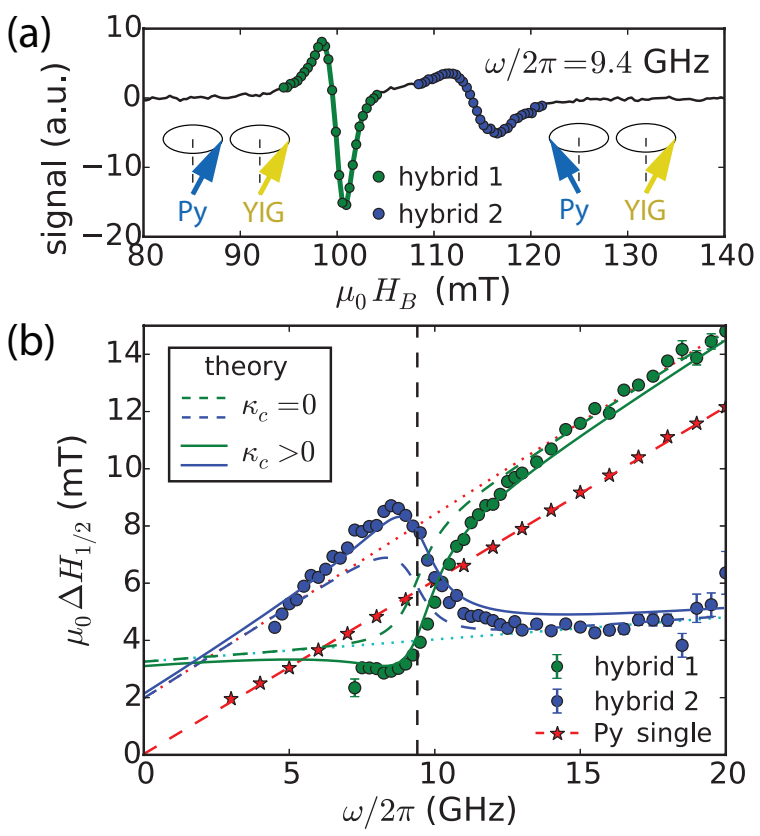

FIG. 2. (a) The lineshape of the Py(7.5 nm)/YIG(100 nm) sample at $\omega / 2 \pi=9.4 \mathrm{GHz}$, showing different linewidths between the two hybrid modes of $\operatorname{YIG}(n=2)$ and $\operatorname{Py}(n=0)$ resonances. (b) Linewidths of the two hybrid modes as a function of frequency. Dotted lines show the linear fit of the linewidths for the two uncoupled modes. Dashed curves show the theoretical values with $\kappa_{c}=0$. Solid curves show the fits with finite $\kappa_{c}$.

when the frequency is near $\omega_{c}$. This is the central result of the paper. It suggests a coherent dampinglike torque which acts along or against the intrinsic damping torque depending on the phase difference of the coupled dynamics of YIG and Py, same as the fieldlike torque acting along or against the Larmor precession.

Because the spin pumping is dissipative, the sign of $\kappa_{c}$ must be positive and the mode with a broader (narrower) linewidth corresponds to the out-of-phase (inphase) precession mode. In Fig. 2(a) the broaderlinewidth mode exhibit a higher resonance field than the narrower-linewidth mode. This is a signature of antiferromagnetic exchange coupling at the YIG/Py interface [25]. From the resonance analysis we also find that all the $\mathrm{SiO}_{2} / \mathrm{Py}$ samples show lower resonance fields than the Py samples grown on YIG [44], which agrees with the antiferromagnetic nature of the YIG/Py interfacial coupling.

To reproduce the data in Fig. 2(b), we introduce the linewidths as the imaginary parts of the resonance fields in Eq. (1):

$$
\begin{aligned}
& \mu_{0}\left(H_{c}^{ \pm}+i \Delta H_{1 / 2}^{ \pm}\right)=\mu_{0} \frac{H_{r}^{\mathrm{YIG}}+H_{r}^{\mathrm{Py}}}{2}+i \mu_{0} \frac{\kappa_{\mathrm{YIG}}+\kappa_{\mathrm{Py}}}{2} \\
& \pm \sqrt{\left(\mu_{0} \frac{H_{r}^{\mathrm{YIG}}-H_{r}^{\mathrm{Py}}}{2}+i \mu_{0} \frac{\kappa_{\mathrm{YIG}}-\kappa_{\mathrm{Py}}}{2}\right)^{2}+\tilde{g}_{c}^{2}}
\end{aligned}
$$

where $\kappa_{\mathrm{YIG}(\mathrm{Py})}$ is the uncoupled linewidth of YIG $(\mathrm{Py})$ from the linear extraction (dotted lines) in Fig. 2(b), and $\tilde{g}_{c}=g_{c}+i \kappa_{c}$ is the complex interfacial coupling strength with an additional dampinglike component $\kappa_{c}$. The dominant mechanism for $\kappa_{c}$ is the mutual spin pumping from the concerted dynamics of YIG and Py [30, 31]. We identify the incoherent spin pumping as the linewidth enhancement of $\mathrm{Py}(7.5 \mathrm{~nm}), \Delta H_{s p}^{\mathrm{Py}}$, away from the avoided crossing, i.e. between the YIG/Py(red dots in Fig. 2b) and $\mathrm{Si} / \mathrm{SiO}_{2} / \mathrm{Py}$ (red stars in Fig. 2b). Then, we quantify the coherent dampinglike coupling strength $\kappa_{c}$ as $\kappa_{c}(\omega)=\beta \mu_{0} \Delta H_{s p}^{\mathrm{Py}}(\omega)$, where $\beta$ is a unitless weight measuring the ratio between the coherent and incoherent spin pumping. For the best fit value, $\beta=0.82$, Eq. (2) nicely reproduces the data in Fig. 2(b). For comparison, if we set $\kappa_{c}=0$ in Eq. (2), we obtain the blue and green dashed curves, which result in identical linewidth at $\omega_{c}$ as opposed to the data in Fig. 2(a).

In order to understand the physical meaning of $\tilde{g}_{c}$, we consider the coupled Landau-Lifshitz-Gilbert (LLG) equations of YIG/Py bilayer [25, 31, 33] in the macrospin limit:

$$
\begin{gathered}
\frac{d \mathbf{m}_{i}}{d t}=-\mu_{0} \gamma_{i} \mathbf{m}_{i} \times \mathbf{H}_{e f f}+\alpha_{i} \mathbf{m}_{i} \times \frac{d \mathbf{m}_{i}}{d t} \\
-\gamma_{i} \mathbf{m}_{i} \times \frac{J}{M_{i} t_{i}} \mathbf{m}_{j}+\Delta \alpha_{i}\left(\mathbf{m}_{i} \times \frac{d \mathbf{m}_{i}}{d t}-\mathbf{m}_{j} \times \frac{d \mathbf{m}_{j}}{d t}\right)
\end{gathered}
$$

where $\mathbf{m}_{i, j}$ is the unit magnetization vector, $\mathbf{H}_{e f f}$ is the effective field including $H_{B}, H_{e x}$ and the demagnetization field, $\alpha_{i}$ is the intrinsic Gilbert damping. The index is defined as $(i, j)=(1,2)$ or $(2,1)$. In the last two coupling terms, $J$ is the interfacial exchange energy and $\Delta \alpha_{i}=\gamma_{i} \hbar g^{\uparrow \downarrow} /\left(4 \pi M_{i} t_{i}\right)$ is the spin pumping damping enhancement. The two terms provide the fieldlike and dampinglike coupling torques, respectively, between $\mathbf{m}_{i}$ and $\mathbf{m}_{j}$. To view the dampinglike coupling on a similar footage, we define its coupling energy $J^{\prime}$ as:

$$
J^{\prime}(\omega)=\frac{g^{\uparrow \downarrow}}{4 \pi} \hbar \omega
$$

Here $J^{\prime}$ describes the number of quantum channels per unit area $\left(g^{\uparrow \downarrow}\right)$ for magnons $(\hbar \omega)$ to pass through [30, 33]; similarly, $J$ describes the number and strength of exchange bonds between YIG and Py per unit area. From the definition, we can express the spin pumping linewidth enhancement as $\mu_{0} \Delta H_{s p}^{i}=J^{\prime} / M_{i} t_{i}$, in pair with the exchange field term in Eq. (3). By solving Eq. (3) we 
find:

$$
\begin{gathered}
\kappa_{i}=\frac{\alpha_{i} \omega}{\gamma_{i}}+\frac{J^{\prime}}{M_{i} t_{i}} \\
g_{c}=f\left(\omega_{c}\right) \cdot \sqrt{\frac{J}{M_{1} t_{1}} \cdot \frac{J}{M_{2} t_{2}}} \\
\kappa_{c}=f\left(\omega_{c}\right) \cdot \sqrt{\frac{J^{\prime}}{M_{1} t_{1}} \cdot \frac{J^{\prime}}{M_{2} t_{2}}}
\end{gathered}
$$

with the dimensionless factor $f(\omega)$ accounting for the precession elliptical asymmetry. $f(\omega)=1$ for identical ellipticity $\left(M_{1}=M_{2}\right)$ and $f\left(\omega_{c}\right)=0.9$ in the case of YIG and Py; See the Supplmental Information for details [44].

Eq. (5) shows that both $g_{c}$ and $\kappa_{c}$ are proportional to $1 / \sqrt{t_{i}}$, which comes from the geometric averaging of the coupled magnetization dynamics. This is in contrast to the $1 / t_{i}$ dependence of the uncoupled exchange field and spin pumping damping enhancement for a single layer, as shown in Eq. (5a). In Fig. 3(a), a good fitting of $g_{c}$ to $1 / \sqrt{t_{\mathrm{Py}}}$ rather than $1 / t_{\mathrm{Py}}$ validates the model. In the limit of zero Py thickness, the model breaks down due to the significance of boundary pinning and the assumption of macrospin dynamics, as reflected in the reduction of $g_{c}$ at $t_{\mathrm{Py}}=5 \mathrm{~nm}$.

For the dampinglike coupling, we plot $\beta$ instead as a function of $t_{\mathrm{Py}}$ because of the variation in the quality of interfacial coupling and the frequency dependence of $\kappa_{c}$. By taking the spin pumping linewidth enhancement as $\mu_{0} \Delta H_{s p}^{\mathrm{Py}}=J^{\prime} / M_{\mathrm{Py}} t_{\mathrm{Py}}$, we obtain the macrospin expression $\beta=0.9 \sqrt{M_{\mathrm{Py}} t_{\mathrm{Py}} / M_{\mathrm{YIG}} t_{\mathrm{YIG}}}$. Fig. 3(b) shows that the extracted $\beta^{2}$ varies linearly with $t_{\mathrm{Py}}$, rather than being independent of it as would be expected for incoherent spin pumping. The fit is not perfect, which may be caused by i) the variation of inhomogeneous broadening of Py in YIG/Py bilayers, or ii) the multi-peak lineshapes in YIG (see YIG $n=0$ lineshapes in Fig. 1b) due to the possible damage during the ion milling process.

If we calculate $\beta$ from the macrospin approximation, the prediction, shown in the red dashed arrow in Fig. 3(b), differs significantly from the experimental data. To account for the difference, we consider a spin wave model for the YIG/Py bilayer, where finite wavenumbers exist in both layers and are determined from the boundary condition [45]. For simplicity, we consider free pinning at the two exterior surfaces of YIG and Py and Hoffmann exchange boundary conditions for the interior interface of YIG/Py [46]. From the spin wave model, we find an additional factor of $\sqrt{2}$ in Eqs. (5b) and (5c); see the Supplemental Information for details [44]. This factor arises because the nonuniform profile of the PSSW mode in YIG reduces the effective mode volume by a factor of two compared with the uniform mode. A similar effect has been previously discussed in spin pumping from PSSW modes [47, 48]. In Fig. 3(b) the theoretical calculation from the spin wave model (cyan dashed arrow) is close to the experimental values. This is an additional
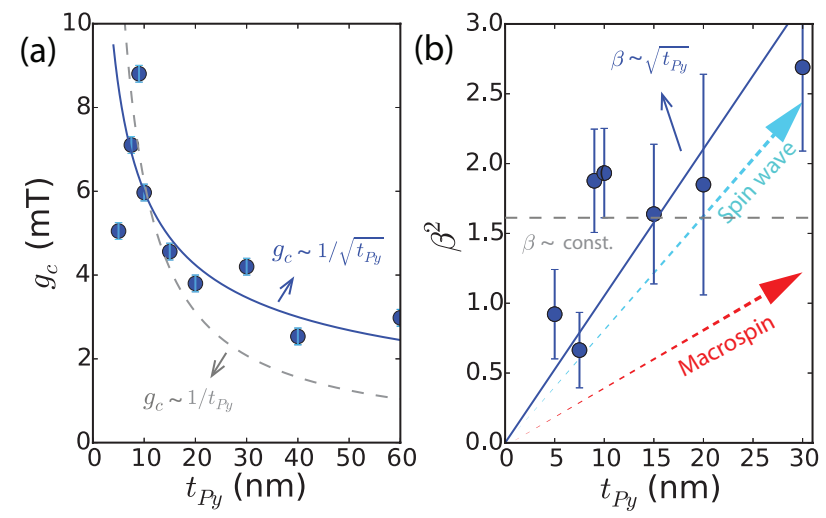

FIG. 3. (a) Extracted $g_{c}$ as a function of $t_{P y}$. (b) Extracted $\beta^{2}$ as a function of $t_{P y}$. In both figures, the solid and dashed curves are the fits of data to the coherent and incoherent models, respectively. In (b), the red and cyan dotted arrows show the theoretical predictions for the coherent models based on the macrospin and spin wave approximations, respectively. Error bars indicate single standard deviations found from the fits to the lineshape.

evidence of the coherent spin pumping in YIG/Py bilayers.

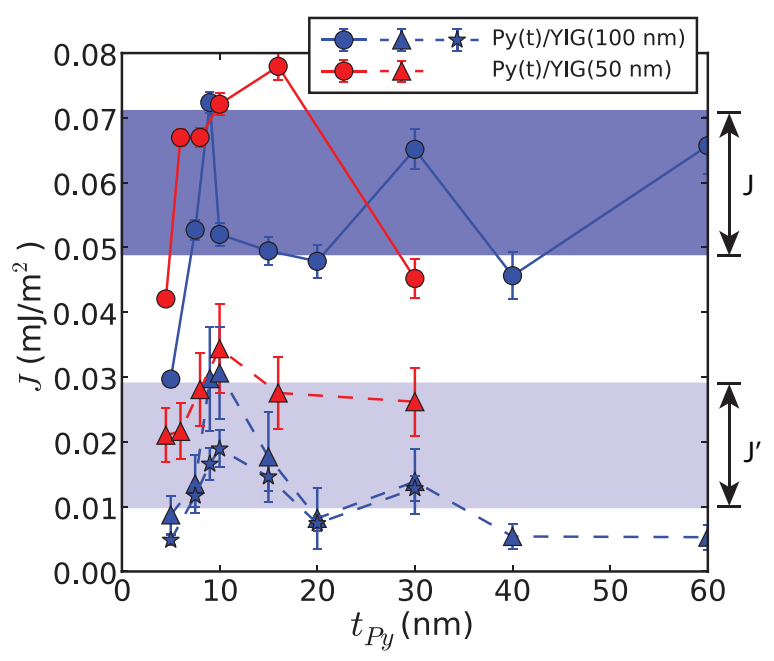

FIG. 4. Thickness dependence of $J$ (circles) and $J^{\prime}$ (triangles), which are calculated from $g_{c}$ and $\kappa_{c}$, respectively. Blue points denote the results for Py/YIG(100 nm) and red points for Py/YIG(50 nm). The blue stars are calculated by the linewidth enhancement between Py/YIG(100 nm) and Py. Error bars indicate single standard deviations found from the fits to the lineshape.

Fig. 4 compares the values of $J$ and $J^{\prime}$ obtained from the hybrid dynamics. For convenience we estimate the value of $J^{\prime}$ at $\omega_{c}$ from Eq. (5c), as $J^{\prime}\left(\omega_{c}\right)=\kappa_{c}\left(\omega_{c}\right) / f\left(\omega_{c}\right)$. $\sqrt{M_{\mathrm{YIG}} t_{\mathrm{YIG}} M_{\mathrm{Py}} t_{\mathrm{Py}} / 2}$, where $\kappa_{c}\left(\omega_{c}\right)$ is half the linewidth difference of the two hybrid modes at $\omega_{c}$ (Fig. 2a). We can also calculate $J^{\prime}$ from uncoupled spin pumping effect, as $J_{s p}^{\prime}=\mu_{0} \Delta H_{s p}^{\mathrm{Py}}\left(\omega_{c}\right) \cdot M_{\mathrm{Py}} t_{\mathrm{Py}}$. For the YIG/Py interface, the value of $J$ stays at the same level; the 
value of $J^{\prime}$ fluctuates with samples but is well aligned with $J_{s p}^{\prime}$, which again supports that the dampinglike interfacial coupling comes from spin pumping. Furthermore, we have also repeated the experiments for a thinner YIG(50 nm)/Py $(\mathrm{t})$ sample series and obtained similar values of $J$ and $J^{\prime}$, as shown in Fig. 4.

Table I summarizes the values of $J, J^{\prime}$ and $g^{\uparrow \downarrow}$ for YIG/Py interface, where $g^{\uparrow \downarrow}$ is calculated from Eq. (4). The value of $J$ is much smaller than a perfect exchange coupled interface, which is not surprising given the complicated and uncharacterized nature of the YIG/Py interface. For Py, the interfacial exchange energy can be estimated [45] by $2 A_{e x} / a$, where for Py $A_{e x}=12 \mathrm{pJ} / \mathrm{m}$ [48] and the lattice parameter $a=0.36 \mathrm{~nm}$. We find $2 A_{e x} / a=68 \mathrm{~mJ} / \mathrm{m}^{2}$, three orders of magnitude larger than $J$. Comparing with similar interfaces, our reported $J$ is similar to YIG/Ni $\left(0.03 \mathrm{~mJ} / \mathrm{m}^{2}[26]\right)$ and smaller than YIG/Co $\left(0.4 \mathrm{~mJ} / \mathrm{m}^{2}[25]\right)$. A different interlayer exchange coupling from Ruderman-Kittel-Kasuya-Yosida interaction may generate a larger $J[49-51]$ but a smaller $g^{\uparrow \downarrow}[52]$. There could also be a fieldlike contribution of $J$ from $g^{\uparrow \downarrow}[25,53-56]$. But since the exchange $J$ dominates in the coupled dynamics, it is difficult to distinguish the spin mixing conductance contribution in our experiments.

\begin{tabular}{cccc}
\hline \hline & $J\left(\mathrm{~mJ} / \mathrm{m}^{2}\right)$ & $J^{\prime}\left(\mathrm{mJ} / \mathrm{m}^{2}\right)$ & $g^{\uparrow \downarrow}\left(\mathrm{nm}^{-2}\right)$ \\
\hline YIG/Py & $0.060 \pm 0.011$ & $0.019 \pm 0.009$ & $42 \pm 21$ \\
\hline
\end{tabular}

TABLE I. Interfacial coupling energy and spin mixing conductance for YIG/Py.

In conclusion, we have characterized the dampinglike coupling torque between two exchange-coupled ferromagnetic thin films. By exciting the hybrid dynamics in the strong coupling regime, this dampinglike torque can either increase or suppress the total damping in the out-ofphase or in-phase mode, respectively. The origin of the dampinglike torque is the coherent spin pumping from the coupling magnetization dynamics. Our results reveal new insight for tuning the coherence in magnon-magnon hybrid dynamics and are important for magnon-based coherent information processing.

Work at Argonne on sample preparation was supported by the U.S. DOE, Office of Science, Materials Sciences and Engineering Division, while work at Argonne and National Institute of Standards and Technology (NIST) on data analysis and theoretical modeling was supported as part of Quantum Materials for Energy Efficient Neuromorphic Computing, an Energy Frontier Research Center funded by the U.S. DOE, Office of Science. Work on experimental design at Oakland University was supported by AFOSR under grant no. FA9550-19-1-0254. V.A. acknowledges support under the Cooperative Research Agreement between the University of Maryland and the NIST Center for Nanoscale Science and Technol- ogy, Award No. 70NANB14H209, through the University of Maryland. Work on microwave spectroscopy at Columbia University was supported by NSF under grant NSF-DMR1411160.

*web54@columbia.edu

$\dagger$ Current address: Department of Materials Science and Engineering, University of Illinois at Urbana-Champaign Urbana, IL 61801 Email: axelh@illinois.edu

‡ weizhang@oakland.edu

[1] M. H. Devoret and R. J. Schoelkopf, Science 339, 1169 (2013).

[2] D. D. Awschalom, L. C. Bassett, A. S. Dzurak, E. L. Hu, and J. R. Petta, Science 339, 1174 (2013).

[3] F. J. Dyson, Phys. Rev. 102, 1217 (1956).

[4] A. V. Chumak, V. I. Vasyuchka, A. A. Serga, and B. Hillebrands, Nature Physics 11, 453 (2015).

[5] A. Khitun, M. Bao, and K. L. Wang, IEEE Trans. Magn. 44, 2141 (2008).

[6] T. Schneider, A. A. Serga, B. Leven, B. Hillebrands, R. L. Stamps, and M. P. Kostylev, Appl. Phys. Lett. 92, 022505 (2008).

[7] V. V. Kruglyak, S. O. Demokritov, and D. Grundler, J Phys. D: Appl. Phys. 43, 264001 (2010).

[8] S. Kaka, M. R. Pufall, W. H. Rippard, T. J. Silva, S. E. Russek, and J. A. Katine, Nature 437, 389 (2005).

[9] F. B. Mancoff, N. D. Rizzo, B. N. Engel, and S. Tehrani, Nature 437, 393 (2005).

[10] N. Locatelli, A. Hamadeh, F. Abreu Araujo, A. D. Belanovsky, P. N. Skirdkov, R. Lebrun, V. V. Naletov, K. A. Zvezdin, M. Muñoz, J. Grollier, O. Klein, V. Cros, and G. de Loubens, Sci. Rep. 5, 17039 (2015).

[11] Y. Li, X. de Milly, F. Abreu Araujo, O. Klein, V. Cros, J. Grollier, and G. de Loubens, Phys. Rev. Lett. 118, 247202 (2017).

[12] R. Lebrun, S. Tsunegi, P. Bortolotti, H. Kubota, A. S. Jenkins, M. Romera, K. Yakushiji, A. Fukushima, J. Grollier, S. Yuasa, and V. Cros, Nat. Commun. 8, 15825 (2017).

[13] A. Awad, P. D urrenfeld, A. Houshang, M. Dvornik, E. Iacocca, R. K. Dumas, and J. Åkerman, Nature Phys. 13, 292 (2017).

[14] D. Vodenicarevic, N. Locatelli, F. Abreu Araujo, J. Grollier, and D. Querlioz, Sci. Rep. 7, 44772 (2017).

[15] M. Romera, P. Talatchian, S. Tsunegi, F. Abreu Araujo, V. Cros, P. Bortolotti, J. Trastoy, K. Yakushiji, A. Fukushima, H. Kubota, S. . Yuasa, M. Ernoult, D. Vodenicarevic, T. Hirtzlin, N. Locatelli, D. Querlioz, and J. Grollier, Nature 563, 230 (2018).

[16] H. Huebl, C. W. Zollitsch, J. Lotze, F. Hocke, M. Greifenstein, A. Marx, R. Gross, and S. T. B. Goennenwein, Phys. Rev. Lett. 111, 127003 (2013).

[17] Y. Tabuchi, S. Ishino, T. Ishikawa, R. Yamazaki, K. Usami, and Y. Nakamura, Phys. Rev. Lett. 113, 083603 (2014).

[18] X. Zhang, C.-L. Zou, L. Jiang, and H. X. Tang, Phys. Rev. Lett. 113, 156401 (2014).

[19] M. Goryachev, W. G. Farr, D. L. Creedon, Y. Fan, M. Kostylev, and M. E. Tobar, Phys. Rev. Applied 2, 054002 (2014). 
[20] L. Bai, M. Harder, Y. P. Chen, X. Fan, J. Q. Xiao, and C.-M. Hu, Phys. Rev. Lett. 114, 227201 (2015).

[21] Y. Li, T. Polakovic, Y.-L. Wang, J. Xu, S. Lendinez, Z. Zhang, J. Ding, T. Khaire, H. Saglam, R. Divan, J. Pearson, W.-K. Kwok, Z. Xiao, V. Novosad, A. Hoffmann, and W. Zhang, Phys. Rev. Lett. 123, 107701 (2019).

[22] J. T. Hou and L. Liu, Phys. Rev. Lett. 123, 107702 (2019).

[23] Y. Tabuchi, S. Ishino, A. Noguchi, T. Ishikawa, R. Yamazaki, K. Usami, and Y. Nakamura, Science 349, 405 (2015).

[24] D. Lachance-Quirion, Y. Tabuchi, S. Ishino, A. Noguchi, T. Ishikawa, R. Yamazaki, and Y. Nakamura, Science Advances 3 (2017), 10.1126/sciadv.1603150.

[25] S. Klingler, V. Amin, S. Geprägs, K. Ganzhorn, H. Maier-Flaig, M. Althammer, H. Huebl, R. Gross, R. D. McMichael, M. D. Stiles, S. T. B. Goennenwein, and M. Weiler, Phys. Rev. Lett. 120, 127201 (2018).

[26] J. Chen, C. Liu, T. Liu, Y. Xiao, K. Xia, G. E. W. Bauer, M. Wu, and H. Yu, Phys. Rev. Lett. 120, 217202 (2018).

[27] H. Qin, S. J. Hämäläinen, and S. van Dijken, Sci. Rep. 8, 5755 (2018).

[28] C. Liu, J. Chen, T. Liu, F. Heimbach, H. Yu, Y. Xiao, J. Hu, M. Liu, H. Chang, T. Stueckler, S. Tu, Y. Zhang, Y. Zhang, P. Gao, Z. Liao, D. Yu, K. Xia, N. Lei, W. Zhao, and M. Wu, Nature Commun. 9, 738 (2018).

[29] K. An, V. Bhat, M. Mruczkiewicz, C. Dubs, and D. Grundler, Phys. Rev. Applied 11, 034065 (2019).

[30] Y. Tserkovnyak, A. Brataas, and G. E. W. Bauer, Phys. Rev. Lett. 88, 117601 (2002).

[31] B. Heinrich, Y. Tserkovnyak, G. Woltersdorf, A. Brataas, R. Urban, and G. E. W. Bauer, Phys. Rev. Lett. 90, 187601 (2003).

[32] K. Lenz, T. Toliński, J. Lindner, E. Kosubek, and K. Baberschke, Phys. Rev. B 69, 144422 (2004).

[33] Y. Tserkovnyak, A. Brataas, G. E. W. Bauer, and B. I. Halperin, Rev. Mod. Phys. 77, 1375 (2005).

[34] S. S.-L. Zhang and S. Zhang, Phys. Rev. B 86, 214424 (2012).

[35] V. P. Amin, J. Zemen, and M. D. Stiles, Phys. Rev. Lett. 121, 136805 (2018).

[36] Y. S. Chen, J. G. Lin, S. Y. Huang, and C. L. Chien,
Phys. Rev. B 99, 220402 (2019).

[37] B. F. Miao, S. Y. Huang, D. Qu, and C. L. Chien, Phys. Rev. Lett. 111, 066602 (2013).

[38] P. Hyde, L. Bai, D. M. J. Kumar, B. W. Southern, C.-M. Hu, S. Y. Huang, B. F. Miao, and C. L. Chien, Phys. Rev. B 89, 180404 (2014).

[39] S. Li, W. Zhang, J. Ding, J. E. Pearson, V. Novosad, and A. Hoffmann, Nanoscale 8, 388 (2016).

[40] M. B. Jungfleisch, V. Lauer, R. Neb, A. V. Chumak, and B. Hillebrands, Appl. Phys. Lett. 103, 022411 (2013).

[41] C. Kittel, Phys. Rev. 73, 155 (1948).

[42] C. Herring and C. Kittel, Phys. Rev. 81, 869 (1951).

[43] S. Klingler, A. V. Chumak, T. Mewes, B. Khodadadi, C. Mewes, C. Dubs, O. Surzhenko, B. Hillebrands, and A. Conca, J. Phys. D: Appl. Phys. 48, 015001 (2014).

[44] See the Supplemental Information for details.

[45] B. Hillebrands, Phys. Rev. B 41, 530 (1990).

[46] F. Hoffmann, A. Stankoff, and H. Pascard, J. Appl. Phys. 41, 1022 (1970).

[47] A. Kapelrud and A. Brataas, Phys. Rev. Lett. 111, 097602 (2013).

[48] Y. Li and W. E. Bailey, Phys. Rev. Lett. 116, 117602 (2016)

[49] S. S. P. Parkin, N. More, and K. P. Roche, Phys. Rev. Lett. 64, 2304 (1990).

[50] M. Belmeguenai, T. Martin, G. Woltersdorf, M. Maier, and G. Bayreuther, Phys. Rev. B 76, 104414 (2007).

[51] L. Fallarino, V. Sluka, B. Kardasz, M. Pinarbasi, A. Berger, and A. D. Kent, Appl. Phys. Lett. 109, 082401 (2016).

[52] H. Yang, Y. Li, and W. E. Bailey, Appl. Phys. Lett. 108, 242404 (2016).

[53] W. Zhang, M. B. Jungfleisch, F. Freimuth, W. Jiang, J. Sklenar, J. E. Pearson, J. B. Ketterson, Y. Mokrousov, and A. Hoffmann, Phys. Rev. B 92, 144405 (2015).

[54] J. Sklenar, W. Zhang, M. B. Jungfleisch, W. Jiang, H. Chang, J. E. Pearson, M. Wu, J. B. Ketterson, and A. Hoffmann, Phys. Rev. B 92, 174406 (2015).

[55] T. Nan, S. Emori, C. T. Boone, X. Wang, T. M. Oxholm, J. G. Jones, B. M. Howe, G. J. Brown, and N. X. Sun, Phys. Rev. B 91, 214416 (2015).

[56] L. Zhu, D. C. Ralph, and R. A. Buhrman, Phys. Rev. Lett. 123, 057203 (2019). 